\title{
Long-Term Outcomes Comparing Minimally Invasive Mitral Valve Repair versus Conventional Mitral Valve Surgery
}

\author{
Matthew Jackson 1*, Manraj S. Sandhu1 ${ }^{1}$, Chao Dong², Bilal Bawamia1, Muhammad Qureshi², \\ Khalid Khan', Andrew Goodwin'1, Simon Kendall', Steven Hunter', Richard Graham1, \\ Jeetendra Thambyrajah'1, Enoch F. Akowuah' ${ }^{1}$
}

${ }^{1}$ Cardiothoracic Surgery, The James Cook University Hospital, Middlesbrough, UK

${ }^{2}$ Cardiology, The Freeman Hospital, Newcastle, UK

Email: *manraj.sandhu1@nhs.net

How to cite this paper: Jackson, M., Sandhu, M.S., Dong, C., Bawamia, B., Qureshi, M., Khan, K., Goodwin, A., Kendall, S., Hunter, S., Graham, R., Thambyrajah, J. and Akowuah, E.F. (2018) Paper Title. World Journal of Cardiovascular Surgery, 8, 127-139. https://doi.org/10.4236/wjcs.2018.88012

Received: June 22, 2018

Accepted: July 31, 2018

Published: August 3, 2018

Copyright $\odot 2018$ by authors and Scientific Research Publishing Inc. This work is licensed under the Creative Commons Attribution International License (CC BY 4.0).

http://creativecommons.org/licenses/by/4.0/

\begin{abstract}
Objectives: To compare the long term outcomes between minimally invasive mitral valve repair (MiMVR) and conventional surgery. Current retrospective comparisons between the techniques frequently report echocardiographical (echo) outcomes early after surgery and rarely report them later. Methods: Patients were selected for MiMVR by the surgical multi-disciplinary meeting from June 2008-March 2013. Patients included had at least two transthoracic post-operative echocardiograms. Echocardiographic parameters including left ventricular size and systolic function, degree of mitral regurgitation (MR) and mean mitral valve gradient were recorded. Clinical outcomes including all-cause mortality, re-operation, recurrence of at least moderate MR and elevated mean mitral valve gradients $>5 \mathrm{mmHg}$ were recorded and compared using Kaplan-Meier survival analysis. Results: 223 patients were screened, 96 (43\%) met the criteria and were included. Thirty-seven patients underwent conventional surgery and 59 underwent MiMVR. Mean clinical follow-up was 6.3 years and echo follow up was 3.2 years. There was a significantly higher recurrence of moderate MR in the conventional group $(38 \%(\mathrm{n}=19)$ versus $17 \%(n=10))$. The mean LV end-diastolic diameter was $4.8 \mathrm{~cm}$ (conventional) versus $5.0 \mathrm{~cm}$ (MiMVR). The incidence of elevated PG was 26\% (n $=13$, conventional $)$ and $23 \%(\mathrm{n}=14$, MiMVR). There was no significant difference in incidence in re-operation (conventional 12\% $(\mathrm{n}=6)$, MiMVR $8.3 \%(\mathrm{n}=$ $5)$ ). Long-term mortality was higher in the conventional group (1.7\% vs. $18 \%$ $\mathrm{p}=0.004)$ although the logistic Euroscore was significantly higher $6.8 \% \pm 5.4$ vs. $3.6 \% \pm 1.6$. Conclusions: Minimally invasive mitral valve surgery is safe and feasible in selected patients with good medium and long-term echocar-
\end{abstract}


diographic follow-up.

\section{Keywords}

Mitral Valve Surgery, Minimally Invasive Surgery, Echocardiogram, Clinical Outcomes

\section{Introduction}

Left untreated, severe mitral regurgitation (MR) carries a poor prognosis. In asymptomatic patients, estimated 5-year rates of death from any cause, death from cardiac causes and adverse events (death, heart failure or new atrial fibrillation (AF)) are 22\%, 14\%, and 33\% respectively [1]. Mitral valve repair (MVR) is the optimal treatment for severe MR caused by degenerative disease carrying lower peri-operative mortality, improved survival, better preservation of left ventricular function and lower long-term mortality [2]-[7].

The first video-assisted minimally invasive mitral valve repair via a lateral thoracotomy was reported in 1996 [8]. Such "keyhole" techniques were pioneered to reduce postoperative pain, blood loss, hospital stays and cost compared to the conventional median sternotomy approach [9] [10]. In Europe and America, it has since been adopted as the standard of care. However, the International Society of Minimally Invasive Cardiac Surgery published a consensus document in 2010 highlighting the lack of data directly comparing the two techniques, and strongly recommended more studies designed to help determine whether minimally invasive mitral valve repair (MiMVR) is better, worse or the same as conventional surgery with respect to important clinical outcomes [11]. We compare clinical and echocardiographic follow-up of our real-world experience of conventional approach versus MiMVR in consecutive patients at a single centre.

\section{Methods}

\subsection{Patient Selection and Follow-Up}

The James Cook University Hospital (JCUH), Middlesbrough, is a large cardiothoracic tertiary referral centre accepting referrals from across the North East of England as well as from distant referral centres across the United Kingdom. Approximately 120 mitral valve repairs are performed per year.

All patients undergoing elective mitral valve repair without coronary artery bypass grafting between June 2008 and March 2013 were considered for inclusion into our study. Patients underwent conventional or minimally invasive surgery following assessment of their mitral valve and surgical technique was decided by the surgical team. Contraindications to a minimally invasive approach were a heavily calcified immobile annulus, infective endocarditis, aortic regurgitation, a dilated aorta precluding use of endo-balloon to occlude the aorta, previous right sided thoracic surgery and known severe peripheral vascular disease. 
Urgent and endocarditis cases were excluded from this study.

The operative technique for MiMVR used at our centre has already been described in detail by Akowuah et al. [12]. All cases were performed via a $5-6 \mathrm{~cm}$ antero-lateral minithoracotomy, used to enter the thorax through the third or fourth intercostal space. Mitral valve repair was performed using a combination of techniques including triangular resection, anterior neochordae and transfer of native chords. A complete or partial annuloplasty ring was always employed. Conventional mitral valve repair was performed using conventional surgical techniques decided by the individual surgeon. Patients who required an intraoperative change in strategy from repair to replacement or intra-aortic balloon counterpulsation therapy were excluded.

A cardiac database prospectively recorded data on all mitral valve operations performed during this time period, including peri-operative and postoperative details and events. Data was collected from this database. Further details were obtained from thorough review of individual case notes, echocardiograms obtained from both JCUH and the referring hospitals and discharge letters.

Patients were required to have a transthoracic echocardiogram performed within two months of the initial operation (the "baseline" post-operative scan); this was commonly performed on the ward prior to discharge. At least one further transthoracic echocardiogram, usually performed at JCUH at the time of their cardiothoracic follow-up appointment, was required to be included in the study. Patients were then subsequently followed up by the referring hospital undergoing echocardiography as per local protocol.

\subsection{Definitions}

All chamber dimensions were defined as normal or mild, moderate or severely dilated as per the EAE (European Association of Echocardiography) guidelines [13]. Left ventricular function was defined as hyperdynamic, normal or mildly, moderately or severely impaired using the Simpson's Biplane technique if sufficient image quality was obtained to clearly demarcate the myocardial border or visually if there was insufficient image quality.

Mean and peak mitral valve gradients were measured using continuous wave doppler. Mitral regurgitation was graded as normal, trace, mild, mild/moderate, moderate, moderate/severe or severe using ESC guidelines and visual estimation [14].

\subsection{Outcomes}

Mortality outcomes were detected using The United Kingdom Office of National Statistics (ONS) data. Incidences of re-operation on the mitral valve or recurrence of at least moderate mitral regurgitation were also recorded.

\subsection{Statistical Analysis}

Data was analysed using IBM SPSS v 20.0 for PC. Continuous variables have the 
mean and standard deviations quoted and were compared using a two-sample t-test. Categorical variables were compared using $\mathrm{z}$ test for proportions. Kaplan Meier survival analysis was performed for mortality, re-operation and recurrence of at least moderate mitral valve regurgitation with log-rank comparisons performed between cohorts.

As only patients with appropriate echocardiograms were included, there was no missing data for the patients included. There was no indeterminate data that arose, as continuous variables were generated from the same echocardiogram software and classified according to EAE guidelines.

\subsection{Ethics}

This was approved by the local Research and Development team as part of an evaluation of our minimally invasive surgery service.

\section{Results}

A total of 224 elective and urgent/emergency mitral valve operations were performed during the study period (conventional midline sternotomy (118 cases), minimally invasive approach (106 cases)). Of these, 96 elective cases met the criteria for inclusion into the study (conventional midline sternotomy (37 cases), minimally invasive approach (59 cases)). Seventy-five (78\%) cases had three or more post-operative echocardiograms performed. Baseline demographics and surgical procedural data can be seen in Table 1.

Patients in the conventional surgery arm were significantly older (71.0 \pm 9.6 vs. $59.9 \pm 12.0$ years, $\mathrm{p}<0.001)$, had a significantly higher logistic euroscore $(6.8 \% \pm 5.4 \%$ vs. $3.6 \% \pm 1.6 \%, \mathrm{p}<0.001)$ and had higher rates of pre-operative AF (49\% vs. $20 \%, \mathrm{p}=0.004)$.

Mean follow-up for clinical events was $6.3 \pm 2.0$ for conventional surgery and $6.3 \pm 1.4$ years for MiMVR (overall $6.4 \pm 1.7$ years $(0.4-9.2)$ ) (Table 2). There was no 12 -month mortality in either cohort. Six-year mortality was $14 \%$ amongst patients who underwent conventional surgical repair and $1.7 \%$ in patients who underwent minimally mitral valve surgery $(\mathrm{p}=0.004)$. Further mitral valve intervention in 4 cases (12\%) who underwent conventional surgery (recurrent MR (3 cases), worsening coronary disease requiring coronary artery bypass grafting with further MV repair for moderate MR (1 case)). Further mitral valve intervention was performed on 5 (8.3\%) cases who underwent MiMVR (mitral valve endocarditis (1 case), failure of repair with recurrent MR (2 cases), severe mitral stenosis ( 2 cases $)$ ). There was no significant difference between the two cohorts for re-intervention $(\mathrm{p}=0.661)$. The time to re-do surgery was $39.5 \pm$ 21.2 months (10.3 - 60.1) for conventional surgery and $32.1 \pm 18.3$ months (16.6 - 56.8) for MiMVR ( $\mathrm{p}=0.073)$.

Mean echocardiographic follow-up was $3.2 \pm 1.8$ years in the conventional surgery arm and $3.2 \pm 1.7$ years in the minimally invasive arm. At least moderate mitral regurgitation occurred following valve repair in 15 (41\%) of cases due to 
Table 1. Patients' characteristics, pre-operative pathology, operative characteristics. Values are mean $( \pm S D)$ or number (proportion \%).

\begin{tabular}{|c|c|c|c|}
\hline & $\begin{array}{l}\text { Conventional surgery } \\
\qquad(\mathrm{n}=37)\end{array}$ & $\begin{array}{l}\text { Minimally invasive mitral } \\
\text { valve surgery }(\mathrm{n}=59)\end{array}$ & $\mathrm{p}$-value \\
\hline \multicolumn{4}{|l|}{ Baseline demographics } \\
\hline Age (years) & $71.0 \pm 9.6$ & $59.9 \pm 12.0$ & $<0.001$ \\
\hline Female gender & $14(38)$ & $19(32)$ & 0.569 \\
\hline Logistic Euroscore (\%) & $6.8 \pm 5.4$ & $3.6 \pm 1.6$ & $<0.001$ \\
\hline Serum creatinine (umol/l) & $92.2 \pm 21.8$ & $89.5 \pm 21.9$ & 0.559 \\
\hline \multicolumn{4}{|l|}{ Pre-op rhythm } \\
\hline Sinus & $18(49)$ & $45(77)$ & 0.006 \\
\hline Atrial fibrillation & $18(49)$ & $12(22)$ & 0.004 \\
\hline Complete heart block & $1(2.7)$ & 0 & 0.204 \\
\hline Paced & $0(0)$ & $1(1.7)$ & 0.424 \\
\hline \multicolumn{4}{|l|}{ Pre-op ejection fraction } \\
\hline Normal $(>55 \%)$ & $29(78)$ & $36(59)$ & 0.077 \\
\hline Mild (45\% - 55\%) & $0(0)$ & $6(10)$ & 0.046 \\
\hline Moderate $(30 \%-45 \%)$ & $6(16)$ & $16(27)$ & 0.215 \\
\hline Severe $(<30 \%)$ & $2(8.1)$ & $1(1.7)$ & 0.308 \\
\hline \multicolumn{4}{|l|}{ Mitral valve pathology } \\
\hline Degenerative & $27(73)$ & $49(78)$ & 0.238 \\
\hline Rheumatic & $1(2.7)$ & $4(6.7)$ & 0.384 \\
\hline Functional & $5(14)$ & $5(0)$ & 0.430 \\
\hline Congenital & $1(2.7)$ & $0(0)$ & 0.204 \\
\hline Previous endocarditis & $3(8.1)$ & $1(1.7)$ & 0.126 \\
\hline \multicolumn{4}{|l|}{ Peri-operative details } \\
\hline \multicolumn{4}{|l|}{ Additional procedures } \\
\hline None & $35(96)$ & $53(90)$ & 0.412 \\
\hline PFO closure & $1(2)$ & $4(6.7)$ & 0.384 \\
\hline ASD closure & $0(0)$ & $0(0)$ & 1 \\
\hline LV mass biopsy & $1(2)$ & $0(0)$ & 0.204 \\
\hline LA mass excision & $0(0)$ & $1(1.7)$ & 0.424 \\
\hline ASD closure and apical bullectomy & $0(0)$ & $1(1.7)$ & 0.424 \\
\hline Bypass time (mins) & $\begin{array}{c}93.6 \pm 25.3 \\
(50-148)\end{array}$ & $\begin{array}{l}165 \pm 144.9 \\
(79-1230)\end{array}$ & $<0.001$ \\
\hline Cross-clamp time (mins) & $\begin{array}{c}66.2 \pm 16.7 \\
31-111\end{array}$ & $\begin{array}{c}104 \pm 22.9 \\
49-154\end{array}$ & $<0.001$ \\
\hline IABP & 0 & 0 & 0.112 \\
\hline Re-opening for bleeding & $6(12)$ & $3(5)$ & 0.069 \\
\hline \multicolumn{4}{|l|}{ Blood product requirements (units) } \\
\hline $\mathrm{RBC}$ & $1.1 \pm 2.3$ & $0.2 \pm 0.8$ & 0.026 \\
\hline FFP & $0.8 \pm 1.7$ & $0.4 \pm 1.0$ & 0.199 \\
\hline Platelets & $0.3 \pm 0.7$ & $0.0 \pm 0.2$ & 0.015 \\
\hline
\end{tabular}




\section{Continued}

\begin{tabular}{cccc} 
MAZE procedure & & & \\
None & $33(89)$ & $43(73)$ & 0.056 \\
Full MAZE & $1(2.7)$ & $3(5,1)$ & 0.569 \\
Left atrial ablation & $2(5.4)$ & $11(19)$ & 0.066 \\
Pulmonary vein isolation & $1(2.7)$ & $2(3.4)$ & 0.849 \\
Atrial fibrillation & & & \\
Pre-operative & $18(49)$ & $12(20)$ & 0.004 \\
Post-operative & $10(27)$ & $13(22)$ & 0.575 \\
Post-operative LV function & & & \\
Hyperdynamic & $0(0)$ & $0(0)$ & 0.204 \\
Normal & $29(78)$ & $53(88)$ & 0.121 \\
Mild & $6(16)$ & $5(8.5)$ & 0.246 \\
Moderate & $2(5.4)$ & $0(0)$ & 0.072 \\
Severe & $0(0)$ & $1(1.7)$ & 0.424 \\
\hline
\end{tabular}

PFO, patent foramen ovale; ASD, atrial septal defect; LV, left ventricle; LA, left Atrium; IABP, intra-aortic balloon pump; RBC, red blood cells; FFP, Fresh Frozen Plasma. Post-operative LV function criteria as per EAE guidelines [13].

Table 2. Clinical outcomes, post-operative outcomes. Values are mean $( \pm \mathrm{SD})$ or number (proportion \%).

\begin{tabular}{cccc}
\hline & $\begin{array}{c}\text { Conventional surgery } \\
(\mathrm{n}=37)\end{array}$ & $\begin{array}{c}\text { Minimally invasive mitral } \\
\text { valve surgery }(\mathrm{n}=58)\end{array}$ & P-value \\
\hline Mortality & $7(19)$ & $1(1.7)$ & 0.003 \\
Six-year mortality & $5(14)$ & $1(1.7$ & 0.004 \\
Re-operation & $4(11)$ & $5(8.6)$ & 0.715 \\
Mean time to-reoperation (months) & $39.5 \pm 21.2$ & $32.1 \pm 18.3$ & 0.073 \\
Recurrence of moderate MR & $15(41)$ & $10(17)$ & 0.011 \\
$\begin{array}{c}\text { Mean time to recurrence (months) } \\
\text { Elevated mitral mean gradient } \\
\text { immediately post-op }\end{array}$ & $15.1 \pm 15.8$ & $20.4 \pm 13.7$ & 0.086 \\
$\begin{array}{c}\text { Elevated mitral mean gradient at any } \\
\text { point during follow-up period }\end{array}$ & $3(8.1)$ & $7(12)$ & 0.555 \\
\hline
\end{tabular}

MR, mitral regurgitation.

conventional surgery and $10(17 \%)$ of minimally invasive mitral surgery at 15.1 \pm 15.8 vs. $20.4 \pm 13.7$ months $(\mathrm{p}=0.086)$. This difference was statistically significant $(\log$ rank $\mathrm{p}=0.011)$.

A mean mitral valve gradient above $5 \mathrm{mmHg}$ (suggesting a mild degree of mitral stenosis) was found in $3(8.1 \%)$ conventional mitral valve repairs and 7 (12\%) MiMVRs on the post-operative scan $(\mathrm{p}=0.555)$. During echocardiographic follow-up, a further $7 / 34(21 \%)$ patients in the conventional arm and $6 / 52$ (12\%) MiMVR patients developed a mean gradient in excess of $5 \mathrm{mmHg}(\mathrm{p}$ $=0.251)$; as previously presented, two patients developed severe mitral stenosis 
requiring repeat surgical intervention, both in the MiMVR cohort.

Left ventricular function in the immediately post-operative period can be seen in Table 1. During echocardiographic follow up, LV function in patients undergoing conventional surgery deteriorated in 3 patients (8.1\%) (normal to mild ( 1 case), normal to moderate (1 case) normal to severe (1 case)) and normalised in 7 patients (19\%) (mild to normal (6 cases), moderate to normal (1 case)). In the MiMVR surgical arm, LV function deteriorated in 12 patients (20\%) (normal to mild (10 cases), normal to moderate ( 1 case), normal to severe ( 1 case)) and normalised in 5 patients (8.5\%) (mild to normal (5 cases)).

The mean left ventricular end-diastolic diameter (LVEDd) immediately post-operatively was $4.9 \mathrm{~cm} \pm 0.8 \mathrm{~cm}$ in the conventional surgical cohort and 5.0 $\mathrm{cm} \pm 0.6 \mathrm{~cm}$ in the MiMVR cohort $(\mathrm{p}=0.516)$. Over the follow-up period, the mean LVEDd was $4.8 \mathrm{~cm} \pm 0.7 \mathrm{~cm}$ and $4.9 \mathrm{~cm} \pm 0.7 \mathrm{~cm}$ respectively $(\mathrm{p}=0.499)$, representing a reduction of $2.0 \%$ in both cohorts.

Kaplan Meier survival curves for all-cause six-year mortality, re-operation and recurrence of at least moderate mitral regurgitation can be seen in Figures 1-3.

No data outliers were identified and therefore additional analyses were not required.

\section{Discussion}

The key findings of this observational study comparing long-term clinical and

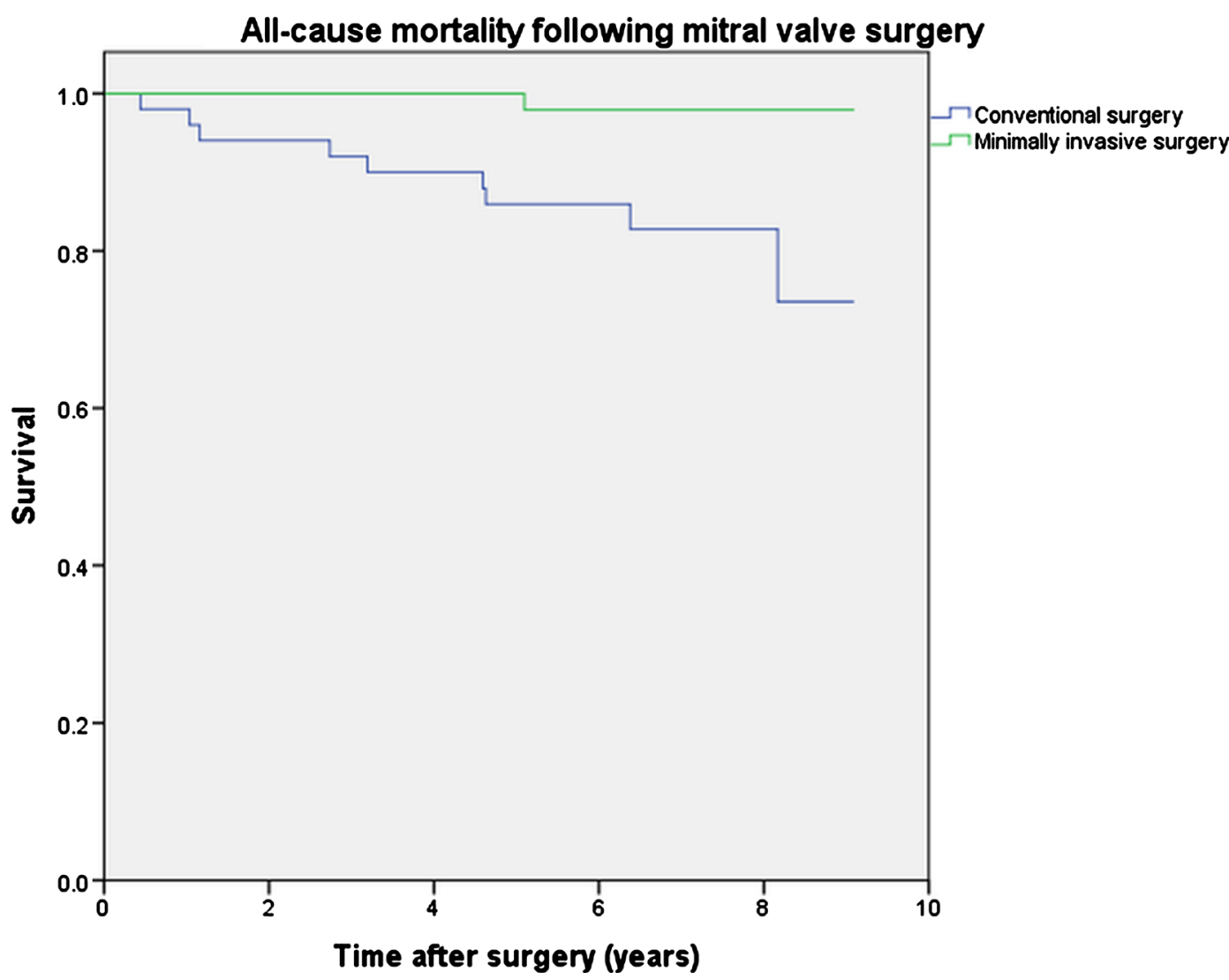

Figure 1. Kaplan Meier survival curve for all-cause six-year mortality following mitral valve repair. 


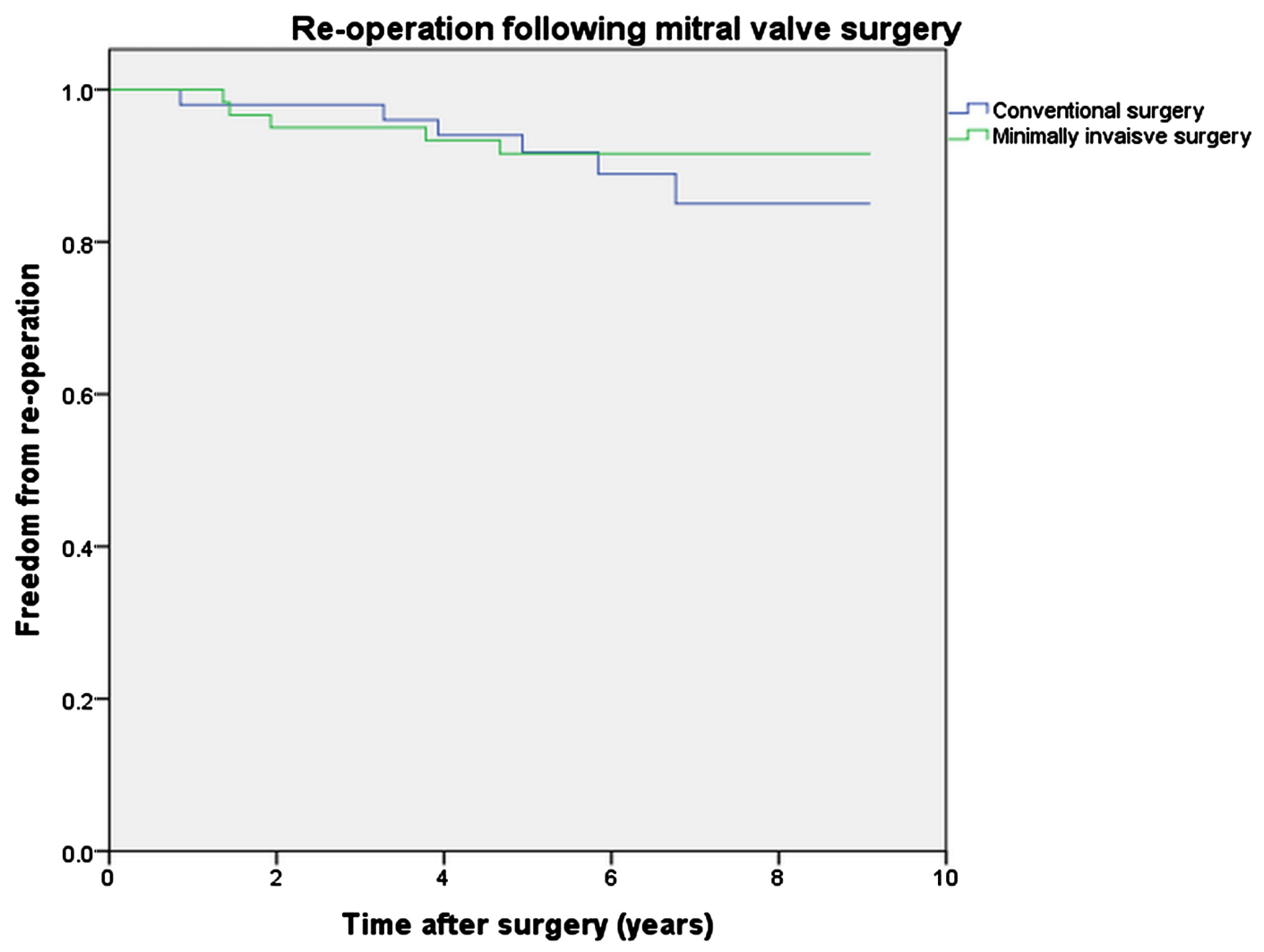

Figure 2. Kaplan Meier survival curve for re-operation following mitral valve repair

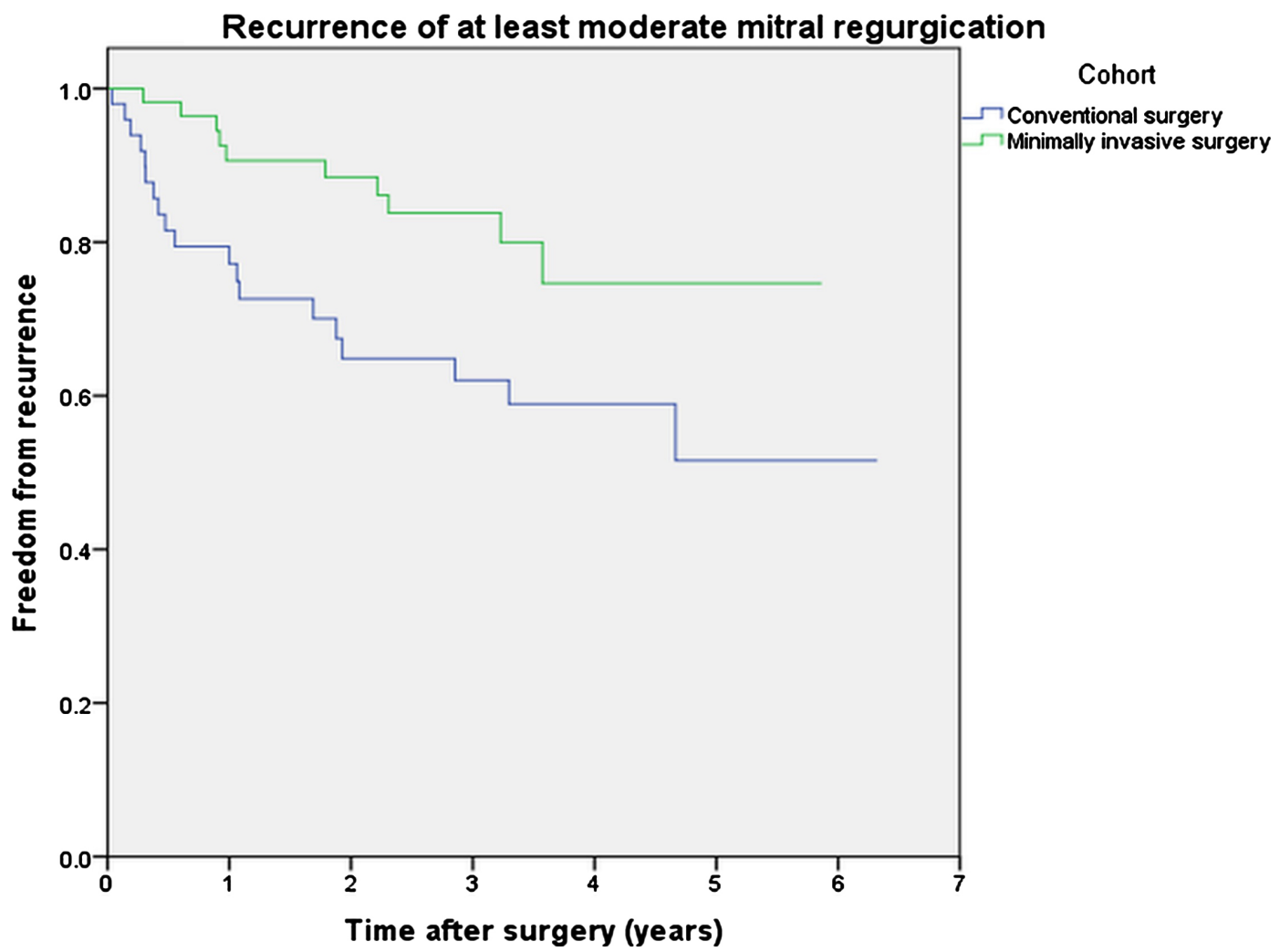

Figure 3. Kaplan Meier survival curve for recurrence of at least moderate mitral regurgitation following mitral valve repair. 
echocardiographic outcomes following conventional and minimally-invasive mitral valve repair are:

1) Mortality and rate of recurrent mitral regurgitation were significantly lower in patients who underwent minimally invasive mitral valve repair.

2) There was no difference between rates of re-operation or elevated postoperative mean mitral valve gradients $>5 \mathrm{mmHg}$.

3) There was no difference in left ventricular end diastolic diameter between the two cohorts.

4) Despite excluding high-risk patients or those undergoing urgent/emergency surgery, patients selected for conventional mitral valve repair remained significantly older with a significantly higher Euroscore reflecting current practice in our centre.

This is the first published study to present both long-term clinical and echocardiographic outcomes comparing conventional and minimally-invasive mitral valve repair in two cohorts recruited in parallel and including multiple aetiologies of mitral regurgitation, mimicking real-world experience. This was performed by the same surgical teams eliminating the potential for variation in experience between centres and operators.

Several meta-analyses compare the surgical outcomes from MiMVR compared to conventional mitral valve repair but do not present echocardiographic follow-up data beyond the immediate post-operative period. Cheng et al. included 35 studies including two small randomised controlled trials (RCTs), showing no benefit in mortality up to 9 years but significant improvement in incidence of atrial fibrillation, chest tube drainage, transfusion requirements, sternal infection, time to return to normal activity and patient scar satisfaction [15]. Luca et al. also showed no difference in mortality despite longer cross-clamp and cardiopulmonary bypass times; rates of sepsis, shorter hospital stays and lower pain levels were lower following MiMVR [16]. Cao et al. note similar findings to the above meta-analyses and comment on the lack of comparative data in the literature [17].

Several studies present long-term echocardiographic follow-up after MiMVR but do not provide comparison data for conventional surgical repair. McClure et al. followed up 1000 patients who underwent minimally invasive mitral valve operations (of which 923 patients were repairs) to a median of 7 years [18]; operative mortality was $0.8 \%$ with re-operation required in $4.4 \%$ of mitral valve repairs. Echocardiographic follow-up was obtained in 615 of 815 eligible mitral valve repairs and demonstrated freedom from recurrent moderate mitral regurgitation at 1,5 and 10 years in $99 \% \pm 1 \%, 87 \pm 2 \%$ and $69 \% \pm 4 \%$ patients. Further, analysis of 707 patients undergoing mitral valve repair demonstrated perioperative mortality of $0.4 \%$, late mortality in 49 (6.9\%) patients and reoperation in $38(4.8 \%)$ patients [19]. Echocardiographic follow-up of 544 patients (mean duration 4.36 years) demonstrated a significant reduction in mean gradient of mitral regurgitation and left ventricular ejection fraction. 
The level of evidence is also limited by the lack of large RCTs in the area. Svensson et al. used propensity matching to compare 590 well-matched patient pairs [20]. In-hospital mortality was similar in both groups $(0.17 \%$ vs. $0.85 \%, p=$ 0.2 ) as was incidence of stroke, renal failure, myocardial infarction and infection; mediastinal drainage and post-operative transfusions were reduced in patients undergoing minimally invasive surgery. However, long-term clinical and echocardiographic outcomes were not reported. The largest RCT compared 140 patients with Barlow's disease repaired using minimally invasive or conventional surgical techniques [21] [22]. Again, there was no difference in mortality, morbidity, freedom from moderate mitral regurgitation or re-operation at 3 years and the trial is limited by the very selective diagnosis of the patients included, the small number of patients studied and the limited detail provided on the primary outcome. Other RCTs also only studied small numbers of patients [23] [24].

The observational nature of our study means there are limitations. Patients were selected by the surgeon for their suitability for a minimally invasive approach, performing conventional surgery on higher-risk patients (urgent/emergency cases, endocarditis). By excluding these high-risk groups, we aimed to minimise this effect; however, patients undergoing conventional surgery were significantly older with a higher logistic euroscore and higher rates of pre-operative atrial fibrillation which may account for the higher event rates experienced during follow-up. Echocardiographic follow-up was not standardised and was determined by local follow-up protocols; some centres performed regular surveillance whilst others performed echocardiography dependent on clinical indications. It is possible that some patients may have developed asymptomatic mitral regurgitation which has therefore not been detected. Finally, patients not followed up in our region were lost to follow-up. Similarly, events that occurred outside of the region may not have been detected; however, patients would have been referred back if re-operation was required and mortality data is collected nationally.

The International Society of Minimally Invasive Cardiac Surgery (ISMICS) consensus document on minimally invasive mitral valve surgery [11] highlights the lack of adequately powered randomised controlled trials comparing the efficacy of the two operations. It concludes that prospective randomised controlled trials, adequately powered to assess post-operative recovery and quality of life, safety (especially stroke rates), efficacy (repair rates) and clinically relevant outcomes, and freedom from re-operation.

In the UK, MiMVR via thoracoscopically-guided right minithoracotomy is limited to a small number of specialist centres and surgeons. Some evidence suggests that MiMVR reduces post-operative complications, hospital stay, and overall recovery time, thus reducing overall costs to the NHS [15] [17] [25] [26] [27] [28] [29]. However, implementing MiMVR would be costly to the NHS, requiring funding to up-skill staff and appropriate infrastructure (equipment etc.). It is therefore imperative that the potential benefits of the approach are 
robustly evaluated to understand whether they outweigh the costs before wide scale adoption is implemented. The ongoing UK Mini Mitral trial funded by the NIHR is designed to assess all of these outcomes and will provide clear and definitive evidence [30].

\section{Conclusion}

Minimally invasive mitral valve repair is a safe and feasible alternative to conventional mitral valve surgery with comparable rates of long-term mortality, re-operation and recurrence of mitral valve regurgitation in a selected cohort of patients. We await the outcomes of the UK Mini Mitral RCT with interest.

\section{Conflict of Interest Statement}

None declared.

\section{References}

[1] Enriquez-Sarano, M., Akins, C.W. and Vahanian, A. (2009) Mitral Regurgitation. Lancet, 373, 1382-1394. https://doi.org/10.1016/S0140-6736(09)60692-9

[2] McNeely, C.A. (2015) Vassileva CM Long-Term Outcomes of Mitral Valve Repair Versus Replacement for Degenerative Disease: A Systematic Review. Current Cardiology Reviews, 11, 157-162.

[3] Gaur, P., Kaneko, T., McGurk, S., Rawn, J.D., Maloney, A. and Cohn, L.H. (2014) Mitral Valve Repair versus Replacement in the Elderly: Short-Term and Long-Term Outcomes. The Journal of Thoracic and Cardiovascular Surgery, 148, 1400-1406.

[4] Coutinho, G.F., Correia, P.M. and Antunes, M.J. (2014) Concomitant Aortic and Mitral Surgery: To Replace or Repair the Mitral Valve? The Journal of Thoracic and Cardiovascular Surgery, 148, 1386-1392.

[5] Candan, O., Ozdemir, N., Aung, S.M., Bakal, R.B., Gecmen, C., Akpinar, S.H., Cem, D., Onur, O. and Kaymaz, C. (2013) Effect of Mitral Valve Repair Versus Replacement on Left Ventricular Rotational Deformation: A Study with Speckle Tracking Echocardiography. The Journal of Heart Valve Disease, 22, 651-659.

[6] Markar, S.R., Sadat, U., Edmonds, L. and Nair, S.K. (2011) Mitral Valve Repair Versus Replacement in the Elderly Population. The Journal of Heart Valve Disease, 20, 265-271.

[7] Chikwe, J., Goldstone, A.B., Passage, J., Anyanwu, A.C., Seeburger, J., Castillo, J.G., Filsoufi, F., Mohr, F.W. and Adams, D.H. (2011) A Propensity Score-Adjusted Retrospective Comparison of Early and Mid-Term Results of Mitral Valve Repair versus Replacement in Octogenarians. European Heart Journal, 32, 618-626.

[8] Mohr, F.W., Falk, V., Diegeler, A., Walther, T., Van Son, J.A. and Autschbach, R. (1998) Minimally Invasive Port-Access Mitral Valve Surgery. The Journal of Thoracic and Cardiovascular Surgery, 115, 567-574.

[9] Seeburger, J., Borger, M.A., Falk, V., Kuntze, T., Czesla, M., Walther, T., Doll, N. and Mohr, F.W. (2008) Minimal Invasive Mitral Valve Repair for Mitral Regurgitation: Results of 1339 Consecutive Patients. European Journal of Cardio-Thoracic Surgery, 34, 760-765. https://doi.org/10.1016/j.ejcts.2008.05.015

[10] Holzhey, D.M., Seeburger, J., Misfeld, M., Borger, M.A. and Mohr, F.W. (2013) Learning Minimally Invasive Mitral Valve Surgery: A Cumulative Sum Sequential 
Probability Analysis of 3895 Operations from a Single High-Volume Center. Circulation, 128, 483-491. ttps://doi.org/10.1161/CIRCULATIONAHA.112.001402

[11] Falk, V., Cheng, D.C.H., Martin, J.P., Diegeler, A., Folliguet, T.A., Nifong, L.B., Perier, P., Raanani, E., Smith, J.M. and Seeburger, J. (2011) Minimally Invasive versus Open Mitral Valve Surgery: A Consensus Statement of the International Society of Minimally Invasive Coronary Surgery (ISMICS) 2010. Innovations. Technology \& Techniques in Cardiothoracic \& Vascular Surgery, 6, 66-76. https://doi.org/10.1097/IMI.0b013e318216be5c

[12] Akowuah, E., Burdett, C., Khan, K., Goodwin, A., Lage, I.B., El-Saegh, M., Smailes, T. and Hunter, S. (2015) Early and Late Outcomes after Minimally Invasive Mitral Valve Repair Surgery. Journal of Heart Valve Disease, 24.

[13] Lang, R.M., Badano, L.P., Mor-Avi, V., Afilalo, J., Armstrong, A., Ernade, L., Flachskampf, F.A., Foster, E., Goldstein, S.A., Kuznetsova, T., Lancellotti, P., Muraru, D., Rietzschel, Er., Rudski, L., Spencer, K.T., Tsang, W. and Voigt, J.U. (2005) Recommendations for Chamber Quantification: A Report from the American Society of Echocardiography's Guidelines and Standards Committee and the Chamber Quantification Writing Group. Developed in Conjunction with the European Association of Echocardiography, a Branch of the European Society of Cardiology. Journal of the American Society of Echocardiography, 18, 1440-1463. https://doi.org/10.1016/j.echo.2005.10.005

[14] Lancelollotti, P., Tribouilloy, C., Hagendorff, A., Popescu, B.A., Edvardsen, T., Pierard, L.A., Badano, L. and Zamorano, J.L. (2013) Recommendation for the Echocardiographic Assessment of Native Valvular Regurgitation: An Executive Summary from the European Association of Cardiovascular Imaging. European Heart Journal-Cardiovascular Imaging, 14, 611-644. https://doi.org/10.1093/ehjci/jet105

[15] Cheng, D.C., Martin, J., Lal, A., et al. (2011) Minimally Invasive versus Conventional Open Mitral Valve Surgery: A Meta-Analysis and Systematic Review. Innovations, 6, 84-103. https://doi.org/10.1097/IMI.0b013e3182167feb

[16] Luca, F., van Garsse, L., Rao, C.M., Parise, O., La Meir, M., Puntrello, C., Rubino, G., Carella, R., Lorusso, R., Gensini, G.F., Maessen, J.G. and Gelsomino, S. (2013) Minimally Invasive Surgery.

[17] Cao, C., Gupta, S., Chandrakumar, D., Nienaber, T.A., Indraratna, P., Ang, S.C., Phan, K. and Yan, T.D. (2013) A Meta-Analysis of Minimally Invasive versus Conventional Mitral Valve Repair for Patients with Degenerative Mitral Disease. Annals of Cardiothoracic Surgery, 2, 693-703.

[18] McClure, R.S., Athanasopoulos, L.V., McGurk, S., Davidson, M.J., Couper, G.S. and Cohn, L.H. (2013) One Thousand Minimally Invasive Mitral Valve Operations: Early Outcomes, Late Outcomes and Echocardiographic Follow-Up. The Journal of Thoracic and Cardiovascular Surgery, 145, 1199-1206. https://doi.org/10.1016/j.jtcvs.2012.12.070

[19] McClure, R.S., Cohn, L.M., Wiegerinck, E., Couper, G.S., Aranki, S.F., Bolman, R.M., Davidson, M.J. and Chen, F.Y. (2009) Early and Late Outcomes in Minimally Invasive Mitral Valve Repair: An Eleven-Year Experience in 707 Patients. The Journal of Thoracic and Cardiovascular Surgery, 137, 70-75. https://doi.org/10.1016/j.jtcvs.2008.08.058

[20] Svensson, L.G., Atik, F.A., Cosgrove, D.M., Blackstone, E.H., Rajeswaran, J., Krishnaswamy, G., Jin, U., Gillinov, A.M., Griffin, B., Navia, J.L., Mihaljevic, T. and Lytle, B.W. (2010) Minimally Invasive versus Conventional Mitral Valve Surgery: A Propensity-Matched Comparison. The Journal of Thoracic and Cardiovascular Surgery, 
39, 926-932. https://doi.org/10.1016/j.jtcvs.2009.09.038

[21] Speziale, G., Nasso, G., Esposito, G., Conte, M., Greco, E., Fattouch, K., Fiore, F., Del Giglio, M., Coppola, R. and Tavazzi, L. (2011) Results of Mitral Valve Repair for Barlow Disease (Bileaflet Prolapse) via Right Minithoracotomy versus Conventional Median Sternotomy: A Randomized Trial. Journal of Thoracic \& Cardiovascular Surgery, 142, 77-83. https://doi.org/10.1016/j.jtcvs.2010.08.033

[22] Nasso, G., Bonifazi, R., Romano, V., Bartolomucci, F., Rosano, G., Massari, F., Fattouch, K., Del Prete, G., Riccioni, G., Del Giglio, M. and Speziale, G. (2014) Three-Year Results of Repaired Barlow Mitral Valves via Right Minithoracotomy versus Median Sternotomy in a Randomized Trial. Cardiology, 128, 97-105. https://doi.org/10.1159/000357263

[23] Dogan, S., Aybek, T., Risteski, P.S., Detho, F., Rapp, A., Wimmer-Greinecker, G. and Moritz, A. (2005) Minimally Invasive Port Access versus Conventional Mitral Valve Surgery: Prospective Randomised Study. The Annals of Thoracic Surgery, 79, 492-498. https://doi.org/10.1016/j.athoracsur.2004.08.066

[24] El-Fiky, M.M., El-Sayegh, El-Beishry, A.S., Abdul Aziz, M., Aboul Enein, H., Waheid, S. and Sallam, I.A. (2000) Limited Right Anterolateral Thoractomy for Mitral Valve Surgery. European Journal of Cardio- Thoracic Surgery, 17, 710-713. https://doi.org/10.1016/S1010-7940(00)00429-2

[25] Sundermann, S.H., Sromicki, J., Rodriguez Cetina Biefer, H., Seifert, B., Holubec, T., Falk, V. and Jacobs, S. (2014) Mitral Valve Surgery: Right Lateral Minithoracotomy or Sternotomy? A Systematic Review and Meta-Analysis. The Journal of Thoracic and Cardiovascular Surgery, 148, 1989-1995.

[26] Ding, C., Jiang, D., Tao, K.Y., Duan, Q.J., Li, J., Kong, M.J., Shen, Z.H. and Dong, A.Q. (2014) Anterolateral Minithoracotomy versus Median Sternotomy for Mitral Valve Disease: A Meta-Analysis. Journal of Zhejiang University Science B, 15, 522-532. https://doi.org/10.1631/jzus.B1300210

[27] Modi, P., Hassan, A. and Chitwood, W.R. (2008) Minimally Invasive Mitral Valve Surgery: A Systematic Review and Meta-Analysis. European Journal of Cardio-Thoracic Surgery, 34, 943-952. https://doi.org/10.1016/j.ejcts.2008.07.057

[28] Goldstone, A.B., Atluri, P., Szeto, W.Y., Trubelja, A., Howard, J.L., MacArthur, J.W., Newcomb, C., Donnelly, J.P., Kobrin, D.M., Sheridan, M.A., Powers, C., Gorman, R.C., Gorman, J.H., Pochettino, A., Bavaria, J.E., Acker, M.A., Hargrove, W.C. and Woo, Y.J. (2013) Minimally Invasive Approach Provides at Least Equivalent Results for Surgical Correction of Mitral Regurgitation: A Propensity-Matched Comparison. Journal of Thoracic \& Cardiovascular Surgery, 145, 748-756. https://doi.org/10.1016/j.jtcvs.2012.09.093

[29] Lamelas, J., Sarria, A., Santana, O., Pineda, A.M. and Lamas, G.A. (2011) Outcomes of Minimally Invasive Valve Surgery versus Median Sternotomy in Patients Age 75 Years or Greater. The Annals of Thoracic Surgery, 91, 79-84.

https://doi.org/10.1016/j.athoracsur.2010.09.019

[30] Akowuah, E., Hancock, H., Maier, R., Kasim, A., Zacharias, J., Deshpande, R., Birdi, I., Vale, L., Livesey, S., Stewart, M. and Murphy, G. (2017) Minimally Invasive Thoracoscopically-Guided Right Minithoractomy versus Conventional Sternotomy for Mitral Valve Repair: A Multicentre Randomised Controlled Trial. http://www.dro.dur.ac.uk/20585 